\title{
Casimir interaction between normal or superfluid grains in the Fermi sea
}

\author{
A Wirzba ${ }^{1}$, A Bulgac ${ }^{2}$, P Magierski ${ }^{2,3}$ \\ ${ }^{1}$ Institut für Kernphysik (Th), Forschungszentrum Jülich, D-52425 Jülich, Germany \\ 2 Department of Physics, University of Washington, Seattle, WA 98195-1560, USA \\ ${ }^{3}$ Faculty of Physics, Warsaw University of Technology, 00-662 Warsaw, Poland \\ E-mail: a.wirzba@fz-juelich.de
}

\begin{abstract}
We report on a new force that acts on cavities (literally empty regions of space) when they are immersed in a background of non-interacting fermionic matter fields. The interaction follows from the obstructions to the (quantum mechanical) motions of the fermions caused by the presence of bubbles or other (heavy) particles in the Fermi sea, as, for example, nuclei in the neutron sea in the inner crust of a neutron star or superfluid grains in a normal Fermi liquid. The effect resembles the traditional Casimir interaction between metallic mirrors in the vacuum. However, the fluctuating electromagnetic fields are replaced by fermionic matter fields. We show that the fermionic Casimir problem for a system of spherical cavities can be solved exactly, since the calculation can be mapped onto a quantum mechanical billiard problem of a point-particle scattered off a finite number of non-overlapping spheres or disks. Finally we generalize the map method to other Casimir systems, especially to the case of a fluctuating scalar field between two spheres or a sphere and a plate under Dirichlet boundary conditions.
\end{abstract}

PACS numbers: 03.65.Nk, 03.65.Sq, 03.75.-b, 21.10.Ma, 74.45.+c

\section{Introduction}

\subsection{The original Casimir effect}

In 1948 the Dutch physicist H.B.G. Casimir predicted the remarkable effect [1] that two parallel, very closely spaced, uncharged metallic plates attract each other in vacuum. The origin of this force can be traced back to the altered (mode sums over the) zeropoint fluctuations of the electromagnetic field which are induced by the presence of the two plates which are added relative to the free case, or rather, which are brought from very far separation into a close distance. The distinctive property of the Casimir effect is that its strength and, perhaps, its sign are geometry-dependent (for a review see [2]). Our aim is to generalize two features of the Casimir effect, the mode sum structure and the geometry-dependence, to the fermionic Casimir effect, where the fluctuating photons are replaced by (non-relativistic) Fermi fields. 


\subsection{Utilizing the geometry dependence of the Casimir energy}

Let us invert the logic and define the Casimir energy as the energy resulting from the geometry-dependent part of the density of states (d.o.s.) - a concept that is closely related to the shell correction energy in nuclear physics:

$$
\rho(E) \equiv \sum_{E_{k}} \delta\left(E-E_{k}\right)=\rho_{0}(E)+\rho_{\text {bulk }}(E)+\delta \rho_{\mathrm{C}}(E, \text { geom.-dep. }) .
$$

Here $\left\{E_{k}\right\}$ are the eigenenergies of the modes, $\rho_{0}$ is the d.o.s. of the homogeneous background and $\rho_{\text {bulk }}$ is the bulk d.o.s. that sums up the excluded volume effects, surface contributions and Friedel oscillations caused by each of the obstacles separately. The remaining part $\delta \rho_{\mathrm{C}}$ is of central interest to us. It is the only term which "knows" about the relative geometry-dependence of the obstacles. Now the Casimir energy can be extracted from the geometry-dependent part of the density of states as a simple integral

$$
\mathcal{E}_{\mathrm{C}} \equiv \int d E E \delta \rho_{\mathrm{C}}(E, \text { geom.-dep. })=-\int d E \mathcal{N}_{\mathrm{C}}(E, \text { geom.-dep. })
$$

where $\mathcal{N}_{\mathrm{C}}(E)$ is the geometry-dependent part of the integrated density of states or number of states, $\mathcal{N}(E) \equiv \sum_{E_{k}} \Theta\left(E-E_{k}\right)=\int_{0}^{E} d E^{\prime} \rho\left(E^{\prime}\right)$.

\subsection{Generalization of the Casimir energy concept to matter fields}

Let us assume that space is not "filled" with fluctuating electromagnetic modes, but with a gas of non-interacting (non-relativistic) fermions. Under this scenario we have the following similarities with the ordinary Casimir effect: In both cases, there exist mode sums, $\sum_{k} \hbar \omega_{k}$, with constant degeneracy factors. The constant weight of the fluctuating electromagnetic modes can be traced back to the two helicity states of the photon. The constancy in the degeneracy of the fermionic matter modes, on the other hand, follows from Pauli's exclusion principle, where the pertinent weight can be formulated in terms of spin and isospin factors. However, the Casimir mode summation over matter fields differs from the case of fluctuating fields by the presence of a new independent scale in addition to the geometric scales (e.g. the separation $L$ and area $A$ of the plates), namely by the presence of the Fermi energy (i.e., the chemical potential $\mu$ at zero temperature).

In the following we will consider the case of matter fields (non-relativistic fermions) located in the space between voids or cavities, such that the matter fields will build up a quantum pressure on the voids. Even if we assume that the matter fields are noninteracting and the voids are non-overlapping, an effective interaction between these empty regions of space will still arise in the background of the non-interacting fermionic matter fields, since the cavities - depending on their geometric arrangement - can shield the free movement of the matter modes such that a net change in the mode sum over the Fermi states in the Fermi sea results.

Applications of this scenario exist e.g. for the inner crust of neutron star. With increasing distance from the star's surface (inside the star), the nuclei start to loose neutrons due to the growing pressure and density. Bulgac and Magierski argued that the shell correction energies (in other words the fermionic Casimir energies) of the 
resulting low-density neutron matter in the presence of bubbles (i.e. nuclei) are of the same order of magnitude [3] as the differences between the usually assumed liquid-dropmodel sequence of phases 4]. Thus disordered lattices of bubbles are in competition with the standard sequence of (i) nuclear drops (nuclei), (ii) nuclear rods, (iii) nuclear plates (all three surrounded by a low-density background of neutrons), (iv) tubes, (v) bubbles (both surrounded by the high-density background of nuclear matter), until finally a uniform nuclear matter phase is reached.

An analogous investigation of bubbles inside a Fermi gas background is also of relevance for the inner core of neutron stars. Under the assumption that quark matter does exist and quark droplets can form there, a similar pattern is predicted, with quarkdroplets (bags) inside hadronic matter taking over the role of the embedded nuclei.

Also in the laboratory, the study of the interaction of cavities inside a uniform fermionic background could be of importance [5]. Examples are $\mathrm{C}_{60}$ buckyballs immersed in liquid mercury. The liquid metal itself serves only as a non-rigid neutral background which provides the Fermi gas environment via its conductance electrons, in which the buckyballs "drill" the voids. Another example would be buckyballs in liquid ${ }^{3} \mathrm{He}$ as Fermi gas. Finally, in the future, boson-condensate droplets immersed in dilute atomic Fermi condensates could serve as systems with which the effective interactions of cavities inside a Fermi gas could be studied in the lab.

\section{Casimir calculation mapped onto a scattering problem}

Note that the Casimir calculation for fermionic (non-relativistic) matter fields simplifies enormously, because the presence of a second scale, the chemical potential $\mu=\hbar^{2} k_{F}^{2} / 2 m$ (or the Fermi momentum $k_{F}$ ), provides for a natural $U V$-cutoff, $\Lambda_{U V} \equiv \mu$ (here $m$ is the mass of the fermion). Thus the Casimir energy for fermions between two impenetrable (parallel) planes at a distance $L$ is simply given by the chemical potential times a finite function of the dimensionless argument $k_{F} L, \mathcal{E}_{\mathrm{C}}=\mu F\left(k_{F} L\right)$.

For more complicated geometries, the computations become more and more involved as it is the case for the ordinary electromagnetic Casimir effect. However, Casimir calculations of a finite number of immersed non-overlapping spherical voids or rods, i.e. spheres and cylinders in 3 dimensions or disks in 2 dimensions, are still doable. In fact, these calculations simplify because of Krein's trace formula [6, 7]

$$
\delta \bar{\rho}(E)=\bar{\rho}(E)-\bar{\rho}_{0}(E)=\frac{1}{2 \pi i} \frac{d}{d E} \operatorname{tr} \ln S_{n}(E),
$$

which links the variation in the level density $\delta \bar{\rho}(E)$ (the difference of the total density of states and the background one) to the energy-variation of the phase shift $\frac{1}{2 i} \ln \operatorname{det} S_{n}(E)$ of the $n$-sphere/disk scattering matrix $S_{n}(E)$. Note that the level densities on the left hand side are averaged over an energy-interval larger than the mean-level spacing in the volume $V$ of the entire system in order to match the continuous expression on the right hand side. In this way the Casimir calculation is mapped to the quantum mechanical 
analog of a classical "billiard" problem: the hyperbolic or even chaotic scattering of a point-particle off an assemble of $n$ non-overlapping spheres (or disks) [8, 9, 10, 11, 12, 13.

Moreover, it is now possible to extract the geometry-dependent Casimir fluctuations from the multiple-scattering part of the scattering matrix. In fact, as shown in 11, 12, 13, the determinant of the $n$-sphere/disk S-matrix can be separated into a product of the determinants of the 1 -sphere/disk S-matrices $S_{1}\left(E, a_{i}\right)$, where $a_{i}$ is the radius of the single scatterer $i$, and the ratio of the determinant of the inverse multiscattering matrix $M(k)$ and its complex conjugate:

$$
\operatorname{det} S_{n}(E)=\left\{\prod_{i=1}^{n} \operatorname{det} S_{1}\left(E, a_{i}\right)\right\} \frac{\operatorname{det}\left(M\left(k^{*}\right)\right)^{\dagger}}{\operatorname{det} M(k)} .
$$

For the fermionic Casimir case, the energy $E$ and the wave number $k$ are related as $E=\hbar^{2} k^{2} / 2 m$. When inserted into Krein's formula, the product over the single-scatterer determinants generates just the bulk (or Weyl term) contribution to the density of states

$$
\bar{\rho}_{\text {bulk }}\left(E,\left\{a_{i}\right\}\right) \equiv \sum_{i=1}^{n} \bar{\rho}_{\text {Weyl }}\left(E, a_{i}\right)=\frac{1}{2 \pi i} \frac{d}{d E} \sum_{i=1}^{n} \ln \operatorname{det} S_{1}\left(E, a_{i}\right),
$$

which takes care of the excluded volume terms and the surface terms (including Friedel oscillations). The geometry-dependent part of the d.o.s. is therefore given by a modified Krein equation [5] which is formulated in terms of the (inverse) multi-scattering matrix instead of the full S-matrix

$$
\begin{aligned}
\delta \bar{\rho}_{\mathrm{C}}\left(E,\left\{a_{i}\right\},\left\{\vec{r}_{i j}\right\}\right) & =\bar{\rho}(E)-\bar{\rho}_{0}(E)-\sum_{i=1}^{n} \bar{\rho}_{\mathrm{Weyl}}\left(E, a_{i}\right) \\
& =-\frac{1}{\pi} \operatorname{Im}\left\{\frac{d}{d E} \ln \operatorname{det} M\left[k(E),\left\{a_{i}\right\},\left\{\vec{r}_{i j}\right\}\right]\right\},
\end{aligned}
$$

where $\vec{r}_{i j}$ are the relative separation vectors between the centers of the spheres (or disks). The pertinent Casimir energy can then be read off from the finite integral

$$
\mathcal{E}_{\mathrm{C}}=\int_{0}^{\mu} d E(E-\mu) \delta \bar{\rho}_{C}=-\int_{0}^{\mu} d E \overline{\mathcal{N}}_{\mathrm{C}} .
$$

\section{The calculation}

Equation (66) does allow to simplify the problem, since there exists a close-form expression for the inverse multi-scattering matrix for $n$ spheres (of radii $a_{j}$ and mutual separation $r_{j j^{\prime}}$, labelled by the indices $\left.j, j^{\prime}=1,2, \cdots, n\right)$ in terms of spherical Bessel and Hankel functions, spherical harmonics and 3j-symbols, where $l, l^{\prime}$ and $m, m^{\prime}$ are total angular momentum and pertinent magnetic quantum numbers, respectively [13]:

$$
\begin{aligned}
& M_{l m, l^{\prime} m^{\prime}}^{j j^{\prime}}=\delta^{j j^{\prime}} \delta_{l l^{\prime}} \delta_{m m^{\prime}}+\left(1-\delta^{j j^{\prime}}\right) i^{2 m+l^{\prime}-l} \sqrt{4 \pi(2 l+1)\left(2 l^{\prime}+1\right)} \\
& \quad \times\left(\frac{a_{j}}{a_{j^{\prime}}}\right)^{2} \frac{j_{l}\left(k a_{j}\right)}{h_{l^{\prime}}^{(1)}\left(k a_{j^{\prime}}\right)} \sum_{l^{\prime \prime}=0}^{\infty} \sum_{m^{\prime \prime}=-l^{\prime}}^{l^{\prime}} \sqrt{2 l^{\prime \prime}+1} i^{l^{\prime \prime}}\left(\begin{array}{ccc}
l^{\prime \prime} & l^{\prime} & l \\
0 & 0 & 0
\end{array}\right) \\
& \quad \times\left(\begin{array}{ccc}
l^{\prime \prime} & l^{\prime} & l \\
m-m^{\prime \prime} & m^{\prime \prime} & -m
\end{array}\right) D_{m^{\prime}, m^{\prime \prime}}^{l^{\prime}}\left(j, j^{\prime}\right) h_{l^{\prime \prime}}^{(1)}\left(k r_{j j^{\prime}}\right) Y_{l^{\prime \prime}}^{m-m^{\prime \prime}}\left(\hat{r}_{j j^{\prime}}^{(j)}\right) .
\end{aligned}
$$


The unit vectors $\hat{r}_{j j^{\prime}}^{(j)}$ point from the origin of sphere $j$ (as measured in its local coordinate system) to the origin of sphere $j^{\prime}$. The local coordinate system of sphere $j^{\prime}$ is mapped to the one of sphere $j$ with the help of the rotation matrix $D_{m^{\prime}, \tilde{m}}^{l^{\prime}}\left(j, j^{\prime}\right)$.

For small scatterers the expression of the multi-scattering matrix even simplifies:

$$
M^{j j^{\prime}}(E) \approx \delta^{j j^{\prime}}-\left(1-\delta^{j j^{\prime}}\right) f_{j}^{s}(E) \frac{\exp \left(i k r_{j j^{\prime}}\right)}{r_{j j^{\prime}}}+\mathcal{O}(p \text {-wave }) .
$$

since only $s$-wave scattering is important; i.e., spherical waves modulated by $s$-wave amplitudes $f_{j}^{s}(E)$ propagate between the spheres. The integrated d.o.s. in the case of two small spherical cavities of common radius $a$ and center-to-center separation $r$ is [5]

$$
\mathcal{N}_{\mathrm{C}}^{\mathrm{oo}}(E)=-\frac{1}{\pi} \operatorname{Im} \ln \operatorname{det} M^{\mathrm{oo}}(E) \approx \nu_{\mathrm{deg}} \frac{a^{2}}{\pi r^{2}} \sin [2(r-a) k]+\mathcal{O}\left((k a)^{3}\right)(10
$$

where $\nu_{\text {deg }}$ is the spin/isospin-degeneracy factor. This expression should be compared with the semiclassical approximation that sums up all partial waves

$$
\mathcal{N}_{\mathrm{C}, \mathrm{sc}}^{\mathrm{oo}}(E)=\nu_{\mathrm{deg}} \frac{a^{2}}{4 \pi r(r-2 a)} \sin [2(r-2 a) k] .
$$

In fact, the latter is the leading contribution to Gutzwiller's trace formula [14], namely the non-repeated contribution of the two-bounce periodic orbit between the two spheres, with the action $S_{p o}(k) / \hbar=2(r-2 a) k$ where $2(r-2 a)$ is the length of the geometric path. Note that the semiclassical result is suppressed by a factor of $1 / 4$ in comparison to the small-scatterer one.

As shown in Ref. [5] the semiclassical result is a very good approximation of the full quantum mechanical result calculated from the exact expression (8) of the two-sphere scattering matrix when plugged into the modified Krein formula (6). Therefore the Casimir energy for the two spherical cavities inside a non-relativistic non-interacting fermion background can be approximated in terms of a spherical Bessel function $j_{1}$ as

$$
\mathcal{E}_{\mathrm{C}}^{\mathrm{oo}}=-\int_{0}^{\mu\left(k_{F}\right)} d E \mathcal{N}_{\mathrm{C}}^{\mathrm{oo}}(E) \approx-\nu_{\mathrm{deg}} \mu \frac{a^{2}}{2 \pi r(r-2 a)} j_{1}\left[2(r-2 a) k_{F}\right],
$$

which is valid for $k_{F} a>1$. This expression is long-ranged, i.e. $1 / L^{3}$ with $L=r-2 a$. For the sphere-plate system the Casimir energy reads instead

$$
\mathcal{E}_{\mathrm{C}}^{\mathrm{o} \mid} \approx-\nu_{\mathrm{deg}} \mu \frac{a}{2 \pi(r-a)} j_{1}\left[2(r-a) k_{F}\right],
$$

which scales even as $1 / L^{2}$ with $L=r-a$. Note that in both cases, the two-sphere system or the sphere-plate system, the Casimir energy does not have a fixed sign in contrast to the standard Casimir effect with fluctuating electromagnetic or scalar fields between these obstacles. Instead the sign of the Casimir energy oscillates as function of the action of the two-bounce orbit. Therefore, under increase of the distance between the cavities, the Casimir energy, which starts out to be attractive, can be made repulsive, and under a further increase of this distance, it can become attractive again, with a decreased strength of course. The reason for this new type of behaviour of a Casimir energy is the presence of a new scale in addition to the length scales, namely the chemical potential $\mu$. In fact, the strength of this fermionic Casimir energy scales with the strength of the 
chemical potential and therefore with the UV-cutoff of the theory. Also this behavior distinguishes the fermionic Casimir effect from the standard Casimir effect: the latter is governed by the infrared behavior of the corresponding density of states.

\section{Fermionic Casimir effect between superfluid grains}

A generalization of the fermionic Casimir effect to the case of superfluid cavities immersed in normal fermionic matter is reported in Ref. [15]. The pairing interaction due to the superfluid obstacles leads to enhanced Casimir contributions, because of the dominance of the particle-hole terms over the particle-particle and hole-hole contributions. Semiclassically, this can be explained by the focusing (and only for large separations defocusing) nature of the Andreev reflections [16] (see Fig. 10) in comparison to the defocusing specular reflections at normal circular boundaries. Since the particle-

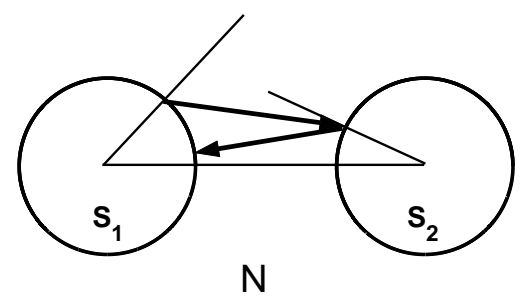

Figure 1. $S_{1}$ and $S_{2}$ are two superfluid grains immersed in a normal Fermi liquid N. Indicated is the Andreev reflection between an incoming particle (hole) and an outgoing hole (particle). The figure is from Ref. [15].

hole d.o.s has the opposite sign to the particle-particle one and since the d.o.s has to be integrated over the quasi-particle energy as measured from the Fermi surface, the resulting Casimir energy for superfluid obstacles is strongly repulsive [15].

\section{Application of the map method to the scalar Dirichlet problem}

Note that the map method onto a scattering problem can be generalized from the Casimir effect in the Fermi sea to other systems; it is especially applicable for the case of a fluctuating scalar field between two spheres or a sphere and a plate (with Dirichlet boundary conditions) [17, 18. Whereas the $s$-wave approximation in the small-scatterer limit is not important for the fermionic Casimir energy which is governed by the UV part of the d.o.s. (i.e. by the contribution at the chemical potential), it is essential for the large distance physics of the fluctuating-scalar Casimir effect, as the latter is governed by the infrared behaviour of the d.o.s. Remember the relative factor of four between the $s$ wave and semiclassical result of the two-cavity d.o.s. The same expressions are valid for the d.o.s. of the fluctuating-scalar Casimir effect. Therefore, the Casimir energy for two far separated Dirichlet spheres is enhanced by a factor of $4 \times\left(90 / \pi^{4}\right)$ [19] relative to the result of the proximity force approximation which is only valid for small separations [20]. The extra $90 / \pi^{4}$ takes into account the missing repeats of the two-bounce orbit in the 
semiclassical formula (11), which are unimportant at large separations, but needed at small ones (see curve D of Fig. 2). For the sphere-plate configuration the corresponding enhancement at large separations is $2 \times 90 / \pi^{4}$, because only one sphere is involved; see Fig. 2 for the comparison of this result (C) with the exact calculation (A) and the $s$-wave approximation (B) [19] and with the proximity force approximations of Refs. [17, 18. The exact data (A) are compatible with numerical data of the wordline approach, within error bars, [17] in the window of overlap, i.e. $L \leq 4 a$. In the case of the electromagnetic

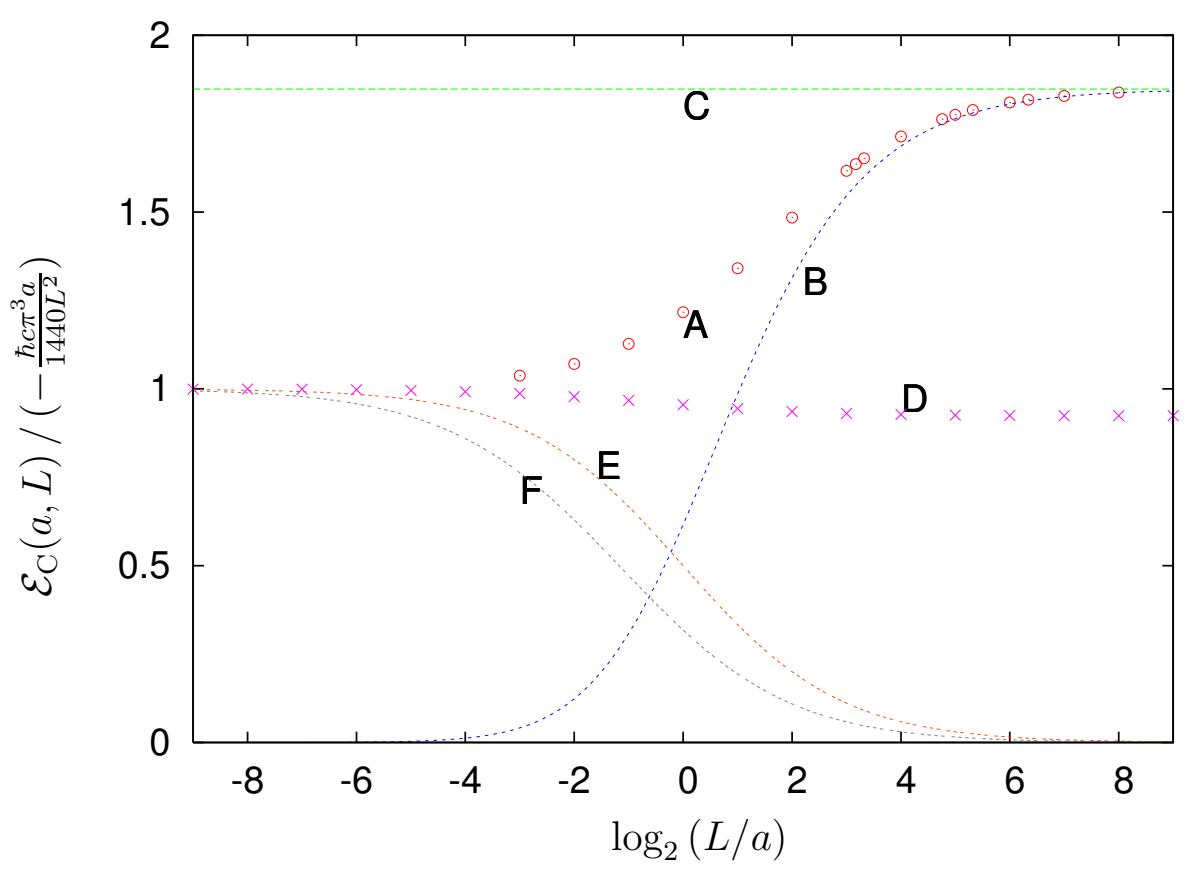

Figure 2. Predictions for the scalar Casimir energy $\mathcal{E}_{\mathrm{C}}(a, L)$ of the Dirichlet sphereplate configuration in units of $-1400 \hbar c \pi^{3} a / L^{2}$ as function of the distance $L$ divided by the radius $a$ of the sphere. The circles (A) represent the numerically calculated exact expression for the sphere-plate system, the curve (B) shows the $s$-wave approximation, the line $(\mathrm{C})$ represents the asymptotic limit $2 \times 90 / \pi^{4}$, the crosses (D) represent the numerically calculated results from the semiclassical Gutzwiller formula including all repeats, see [19]. The curves (E) denotes the plate-based, the curve (F) the spherebased proximity-force approximation, see [17, 18.

Casimir effect, the $s$-wave dominance at large separation has to be replaced by a $p$-wave dominance, since the charge-neutrality of the sphere forbids a monopole term, whereas the standard Casimir-Polder energy is dominated by induced-dipole contributions.

\section{Summary}

We have shown that there exists an effective interaction between voids inside a Fermi gas background, even if the fermions are non-interacting. This new form of Casimir energy is neither attractive nor repulsive, but oscillates according to the relative arrangement of the cavities (in the superfluid case it is strongly repulsive). The spheres can be replaced with other objects, if the curvature radii are larger than the Fermi wave length. The 
effects of finite surface thickness can be booked as Weyl-term contributions and do not affect the geometrical Casimir part if the objects do not overlap. Since the Casimir interaction between bubbles is oscillating and rather long-ranged (in comparison to van der Waals terms), disordered lattices are expected as emerging structures. The disorder can be further enhanced by finite temperature $T$ and corrugated surfaces. The map method can be generalized to other Casimir systems, especially to the scalar Casimir effect for Dirichlet spheres or sphere-plate systems.

\section{Acknowledgments}

A.W. would like to thank Professors Emilio Elizalde, Sergei Odintsov and Joan Soto for the excellent organization of the Workshop QFEXT'05 and Holger Gies and Antonello

Scardicchio for discussions. Support from the Forschungszentrum Jülich under contract No. 41445400 (COSY-067), from the Department of Energy under grant DE-FG0397ER41014, and from the Polish Committee for Scientific Research (KBN) under Contract No. 1 P03B 05927 is gratefully acknowledged.

\section{References}

[1] Casimir H B G 1948 Proc. K. Ned. Akad. Wet. 5179

[2] Bordag M, Mohideen U and Mostepanenko V M 2001 Phys. Rept. 3531

[3] Bulgac A and Magierski P 2001 Nucl. Phys. A 683 695; Bulgac A and Magierski P 2002 Erratum: Nucl. Phys. A 703 892; Bulgac A and Magierski P 2001 Phys. Scr. T90 150

[4] Baym G, Bethe H A and Pethick C 1971 Nucl. Phys. A 175 225; Ravenhall D G, Pethick C J and Wilson J R 1983 Phys. Rev. Lett. 502066

[5] Bulgac A and Wirzba A 2001 Phys. Rev. Lett. 87120404

[6] Krein M G 1953 Mat. Sborn. (N.S.) 33 597; Krein M G 1962 Sov. Math.-Dokl. 3 707; Birman M Sh and Krein M G 1962 Sov. Math.-Dokl. 3740

[7] Beth E and Uhlenbeck G E 1937 Physica 4 915; Huang K 1987 Statistical Mechanics, John Wiley \& Sons, New York, ch. 10.3; Friedel J 1958 Nuovo Cim. Ser. 10 Suppl. 7 287; these results for the correction to the density of states are particular cases of the Krein formula 6 .

[8] Eckhardt B 1987 J. Phys. A: Math. Gen. 205971

[9] Gaspard P and Rice S A 1989 J. Chem. Phys. 90, 2225; 90, 2242; 90, 2255

[10] Cvitanović P and Eckhardt B 1989 Phys. Rev. Lett. 63823

[11] Wirzba A 1999 Phys. Rept. 3091

[12] Wirzba A and Henseler M 1998 J. Phys. A: Math. Gen. 312155

[13] Henseler M, Wirzba A and Guhr T 1997 Ann. Phys., NY 258286

[14] Gutzwiller M C 1990 Chaos in Classical and Quantum Mechanics (Springer, New York)

[15] Bulgac A, Magierski P and Wirzba A 2005 Europhys. Lett. 72, 327

[16] Andreev A F 1964 Zh. Eksp. Teor. Phys. 46 1823; de Gennes P G 1998 Superconductivity of Metals and Alloys, (Addison-Wesley, Reading, MA); Blonder G E et al 1982 Phys. Rev. B 254515

[17] Gies H, Langfeld K and Moyaerts L 2003 JHEP 0306018

[18] Jaffe R L and Scardicchio A 2004 Phys. Rev. Lett. 92 070402; Scardicchio A and Jaffe R L 2005 Nucl. Phys. B $\mathbf{7 0 4} 552$

[19] Bulgac A, Magierski P and Wirzba A 2005 Preprint hep-th/0511056

[20] Derjaguin B V, Abrikosova I I and Lifshitz E M 1956 Q. Rev. 10 295; Blocki J, Randrup J, Swiateck W J and Tsang C F 1977 Ann. Phys., NY 105427 